During first 7 years after the accident (1986-1992) we did not find any increase of ChAL incidence in the whole group of children (0-14 year old) (RR 1.03; 95\% CI 0.92 to 1.14; $\mathrm{p}=0.61)$. However, the IR of infant AL (0-1 year old) increased significantly in this period-from 49 (IR 4.33) before Chernobyl to 67 cases (IR 6.36) in 1986-1992 (RR 1.47; $\mathrm{p}=0.04$ ). Older age groups did not show any increase in ChAL rates.

Following 7-years period (1993-1999) revealed the statistically significant decrease of incidence of infant leukaemia: from 49 (IR 4.33) before Chernobyl to 16 cases (IR 2.29) in 1993-1999 years (RR $0.53 ; \mathrm{p}=0.024)$.

During the following period (2000-2006) we found a further decrease of the incidence of infant leukaemia with only 3 cases (IR 0.47 ) in 7 years. It is highly significant when compared with 49 cases (IR 4.33) before Chernobyl ( $p=0.0000053, R R=0.11)$.

The carcinogenic effects of low dose radiation exposure may be restricted to children exposed in utero or in early infancy $(0-12$ months) during the first years after explosion. Following after dramatic decrease of IRs of infant leukaemia might be explained by the developing of adaptive response to chronic low dose ionising radiation exposure.

\section{2-1.5 CERVICAL AND BREAST CANCER IN LATIN AMERICA: A NEOPLASTIC TRANSITION}

doi:10.1136/jech.2011.142976a.50

${ }^{1} \mathrm{~S}$ Lewis, ${ }^{* 1} \mathrm{H}$ Gómez Dantés, ${ }^{2} \mathrm{R}$ Lozano. ${ }^{1}$ National Institute of Public Health, Cuernavaca, Morelos, Mexico; ${ }^{2}$ Institute for Health Metrics and Evaluation, Seattle, Washington, USA

Introduction Demographic and epidemiologic transitions in Latin American and Caribbean countries have contributed to shifts in the main types of cancers that affect women, characterised by differential burdens of cancer associated with a country's stage of demographic transition.

Methods We compiled statistics from literature indexes, registries and databases on breast and cervical cancer morbidity, mortality, and disability-adjusted life years for 28 LAC countries. Incidence was analysed over time and mortality and DALYs were analysed descriptively and comparatively to arrive at proportional burdens of each cancer. Case-fatality ratios were calculated and plotted against single-year incidence rates.

Results Countries further advanced in their demographic transitions have a greater magnitude of breast cancer, namely in the anglophone Caribbean, Uruguay, and Argentina. Cervical cancer burden is generally greater in Andean and Central American countries and Haiti, which are in earlier stages of demographic transition. Case- fatality ratios for breast cancer range from 0.28 in the Dominican Republic to 0.49 in Cuba, and decrease as incidence rates increase. Cervical cancer ratios vary between 0.34 in Puerto Rico and Argentina to more than 0.50 in nine LAC countries and tend to increase with increased incidence. Bolivia, Honduras, Guatemala and Haiti have ratios above 0.45 for both cancers.

Conclusions Mortality is unacceptably high for both cancers due to lack of early detection and inadequate resources for effective treatment. Since the risk factors that cause breast cancer are largely unmodifiable and tend to increase with economic development, its share of the burden is likely to grow.

\section{2-1.6 CHILDHOOD, EARLY ADULTHOOD, AND MIDDLE AGE ADIPOSITY AND RISK OF POSTMENOPAUSAL ENDOMETRIAL CANCER}

doi:10.1136/jech.2011.142976a.51

T 0 Yang, ${ }^{*}$ B Cairns, G Reeves, N Allen, S Sweetland, V Beral. Million Women Study, University of Oxford, Oxford, UK

Background Middle age obesity is a common risk factor of postmenopausal endometrial cancer and breast cancer. Recent studies suggest that childhood and early adulthood obesity might be independent protective factors against breast cancer, while this effect has not been well understood in endometrial cancer.

Methods This analysis is based on 378614 postmenopausal British women in the Million Women Study who reported validated information of their body shape at 10 years old, clothes size at 20 years old, current body mass index, and other information in middle age (mean age 58 years), and follow-up by the National Health Service Central Registers for 6.05 years on average. Women with recent use of hormone replacement therapy were excluded. Cox regression is used to estimate the risk of endometrial cancer.

Results There are significant associations between body size at 10, 20 and middle age. Having a larger body size at 10 years old, at 20 years old or in middle age is each associated with a higher risk of endometrial cancer in middle age. However, the risks associated with body size at 10 and 20 years old are attenuated after adjustment for or stratification with middle-aged body size.

Conclusion The association of postmenopausal endometrial cancer with childhood or early adulthood obesity is largely explained through body size in middle age. The independent effect of childhood or early adulthood body size on postmenopausal endometrial cancer is not seen 